\title{
Some Medicinal Bryophytes: their Ethnobotanical Uses and Morphology
}

\author{
ANDREA G. AZUELO \\ azuelonenecmu@yahoo.com \\ LALAINE G. SARIANA \\ lalainesariana@yahoo.com \\ MELANIE P. PABUALAN \\ melanie_pulvera@yahoo.com \\ Central Mindanao University, \\ Musuan, Bukidnon, Philippines
}

Date Submitted: June 22, 2011

Final Revision Accepted: July 15, 2011

Abstract - Medicinal bryophytes and their morphological features and ethnic uses were examined and documented. Field collections of bryophytes were made through transect walks along the two mountain sites of Bukidnon, namely: Mt. Kalatungan and Mt. Kitanglad. The number of taxa currently determined with medicinal properties was based from the collected species. The species with medicinal uses was generated from a pool of resources, literature review and scientific journals. Description on their morphological characters was made using microscopic examinations. Taxonomic characters used include leaf orientation, stem structure, sporophyte characters and their habit to classify into families, genera and species. Species composition between study sites was also compared. Visual habitat as to habitat preference and identification for each species was employed. Results of the study highlight the difference in the morphological features among species of medicinal bryophytes, their ethnic uses, and adaptations within the forested area. Mt. Kitanglad exhibited seventeen (17) species of medicinal bryophytes. Of these, eleven (11) are mosses belonging to seven (7) families in eleven (11) genera and six (6) liverworts belonging to five (5) families in six (6) genera. Mt. Kalatungan exhibited fourteen 
(14) species of medicinal bryophytes. Of these, eight (8) are mosses belonging to six (6) families in eight (8) genera and six (6) liverworts belonging to five (5) families in six (6) genera. The moss species with medicinal properties belongs to the genera Sphagnum, Polytrichum, Rhodobryum, Fissidens, Bryum, Mnium, Dawsonia, Philonotis, Pogonatum, Barbula and Plagiomnium. The liverworts species include Marchantia, Pallavicinia, Herbertus, Riccardia, Dumortiera and Plagiochila. The species under study exhibited antimicrobial activity, anti-tumor, anti-cancer, antileukemic activity and healing effects based from secondary data.

Keywords - ethnobotany, morphology, bryophytes, taxonomic treatment, floristic, ethnic uses

\section{INTRODUCTION}

Cryptogam studies specifically on bryophytes had been more dynamic due to more extensive inventory and collective activity among the biologists. The bryophytes which comprises the liverworts (Marchantiophyta), with 6,000 species, the hornworts (Anthocerotophyta) with 300 species, and mosses (Bryophyta) with 15,000 species were thought to be the second largest group of land plants after flowering plants and are pivotal in the early land plant evolution (Shaw and Renzalia, 2004). These groups are too small and are characterized by dominant perennial gametophytic stages, with relatively small, unbranched sporophyte that remain permanently attached to the maternal gametophyte (Cox et al. 2010). Recently, they include approximately 24,000 species worldwide and are important components in many forest ecosystems and constitute a major part of the biodiversity in moist environments, wetland, and mountain ecosystems (Hallingback and Hotsgetts 2000).

Further, bryophytes are important environmental indicators and have been used as predictors of past climate change to validate climate models and potential indicators of global warming (Gignac 2001). Reports showed that bryophytes can be important contributors to the total stream metabolism, nutrient cycling, food web interactions in streams and as direct food source for some invertebrates. More 
importantly, some species are of great source for herbal medicine. The Philippine moss flora had been bryologically documented in relation to taxonomic studies. Currently, diversity and ecological studies on bryophytes had been in some forests in Mindanao. As noted, diversity studies in Mt. Malindang (Azuelo 2005), and in Mt. Kitanglad (Azuelo et al. 2007) and Mt. Kalatungan (Azuelo 2005, Lubos 2000) had been recently investigated. In fact, detailed analysis and comparison of the patterns of species diversity and of species-habitat relationships were noted useful in the development of strategies for the conservation of bryophytes (Hallingback and Tan 2010). However, to date there have been no attempts to document Philippine bryophytes as to its medicinal properties, ethnic uses and other valuable uses, thus the present study makes a stronger effort in documenting the bryofloral species inhabiting the forests under study.

Mt. Kalatungan and Mt. Kitanglad are both natural parks situated in Bukidnon. The former has an elevation up to 2,824 masl, and the latter has an elevation up to 2,938 masl. Both forests exhibited vegetation rich on its bryofloral species and showed high diversity patterns due to their habitat conditions and the habitat preference allowed them to grow and display unique morphological features thus reflecting the diversity status of the forests. However, the said forests are threatened by habitat conversion and negative environmental factors hence, there is a need therefore, to help maintain a rich bryophyte flora by conserving the sites and protective measures be given preference before they are in the verge of extinction. Since bryophytes are not well known to the general public, even among some conservationists, it is necessary to continue our effort to highlight the importance of their presence in nature and their beneficial role in the ecosystem and economic importance as medicinal source.

\section{OBJECTIVES OF THE STUDY}

Specifically, the study aimed to provide taxonomic data and morphological adaptations of some bryophytes identified with medicinal properties, assess the bryofloral species composition of the two natural parks, and determine the ethnic uses of medicinal bryophytes. 


\section{MATERIALS AND METHODS}

\section{Survey of the Study Site}

The study was conducted at the two natural parks in Bukidnon namely: Mt. Kalatungan and Mt. Kitanglad. The former is situated at Pangantucan, Bukidnon and is regarded as a rainforest ecosystem with an estimated area of 21,301.34 ha. The range consists of various peaks and is proclaimed as a protected area in March, 2000, while the latter is situated in Impasug-ong, Bukidnon. It consisted with several peaks and its vegetation around its range exhibit richness reflecting the diversity of the vegetation types.

Field survey and collections of bryophytes were done at three different vegetation types. Inventory and assessment of the bryophytes were done by a transect walk (Alpha Taxonomy) for each vegetation type. This was done by listing all the bryophytes seen or collected along the trail. Collection and recording of species was done along a 5-meter area on each side of the transect line. In each site, a 20x20 meter quadrat was established to estimate the bryophyte cover using a calibrated plastic cord.

\section{Preparation of Herbarium Specimen}

The collected specimen of bryophytes were placed in a plastic bags with a field label data: altitude, collection number, collector's name, date of collection, vegetation types, and their ecology and associated habitats. This was then air dried and placed in packet envelope and were properly labeled for herbarium vouchers. The herbaria were deposited at the CMU Museum Botanical Section.

\section{Identification, Classification and Description of Bryophyte Species}

The specimen collected were identified, classified and described morphologically by their diagnostic characters such as habit, habitat, leaf arrangement, stem structure and sporophyte characters (Yamaguchi, 1993). Identification was made using the existing herbaria and keys from books and scientific articles and journals. Further examinations was done through microscopy examinations.

\section{Selection and Identification of Medicinal Bryophytes}

From the collected species, the bryophytes with medicinal 
properties were reassessed and given preference. Knowing their representative taxa, identification of uses was based from different sources such as literature review, scientific journals, on-line journals and website search. The species with potential medicinal value was reexamined using the noted taxonomic treatment and through microscopy analysis on the uniqueness of their structures for none of the recent reviews include a detailed description on its morphological features and habitat preference and habitat associations. Final listing of the medicinal bryophytes collected were highlighted in detail.

The present study likewise focused on the ethnic uses and other economic uses of the medicinal bryophytes. The current data given in the study were all primarily obtained from literature review, and the historical studies. Analysis of distribution patterns of the medicinal species in the two study sites were also noted.

Assessment of conservation status of bryophytes species may be rare, widespread, endemic, threatened or endangered was based on International Union for Conservation of Nature (IUCN) and from scientific journals and websites search. The new Annotated Philippine Moss Checklist by Tan and Iwatsuki (1991) was also used. Local assessment in this study include the rarity and distribution pattern of the species.

\section{Photographs}

Photographs were made from the natural habitat of the species. Information, Education and Communication (IEC) materials were prepared on medicinal species.

\section{Research Ethics}

Research Ethics was observed by getting a gratuitous permit from the Department of Environment and Natural Resources to conducat the study in the sampling sites and permit from the leader of the indigenous people who live in the area.

\section{RESULTS AND DISCUSSION}

A. The Study Area

Mt. Kitanglad and Mt. Kalatungan are situated in Bukidnon, 
Philippines. Both are natural parks, hence were declared a protected areas. The former is a volcanic Lanao-Bukidnon Highland with an area of 32,297 ha and with several peaks, and having an elevation of 2,938 masl, while the latter has an estimated area of 21,301.34 ha. with various peaks and an elevation of 2,824 masl. Both forests displayed natural diversity of its flora and fauna.

\section{B. Taxonomy}

The first phase of the study was a field inventory of the bryophytes flora in the two natural parks in Bukidon namely: Mt. Kitanglad and Mt. Kalatungan. The bryophytes were collected, classified and identified using taxonomic keys, existing herbaria, voucher specimens. Each species was described based habitat, diagnostic characters using hand lens and microscopes. Line drawing of each species is also shown for easy identification (Table 1).

The second phase in the study focused on the medicinal properties exhibited by the bryophytes based on the literature search from pool of resources of scientific journals, articles, online journals and websites (Table 2, 4, and 5).
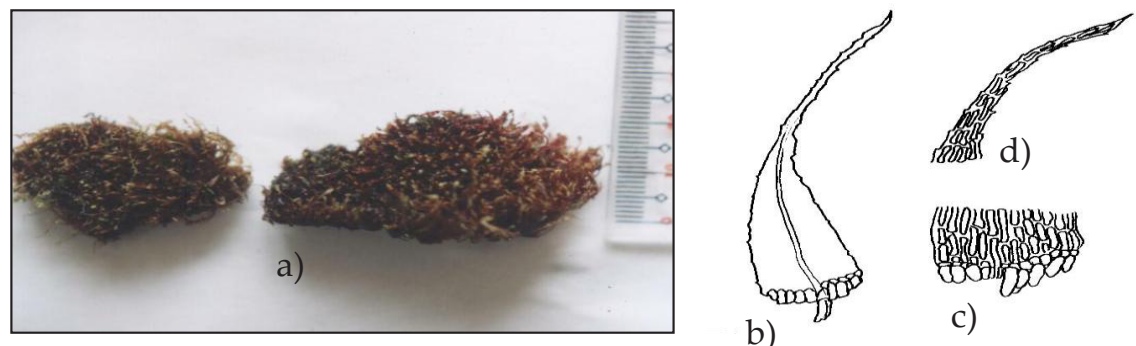

Fig. 1. FAMILY BARTRAMIACEAE

Philonotis sp.1

a) Plant habitat

b) Leaf

c) Basal leaf cells

d) Apical leaf cells; drawn from x 400

Description: Plants slender and erect. Green when fresh and whitish yellow green when dry. Leaves spreading/ patent, oblong ovate, involute leaves. Leaf base plain. Leaf apex setaceous. Leaf 
margin entire. Absence of alar cells. Costa up to apex. Capsule erect, acrocarpous, ovoid, absence of operculum, long seta.

Habitat: on tree trunk
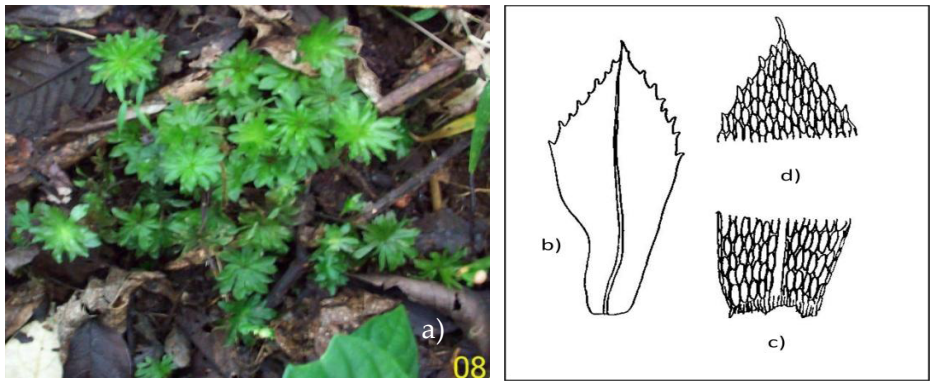

Fig. 2. FAMILY BRYACEAE

Rhodobryum giganteum (Schwaegr.) Par.
a) Plant habit
c) Basal leaf cells
b) Leaf
d) Apical leaf cells; drawn from x 50

Description: Plants large, forming tufts. Dark green when fresh and green when dry. Leaves spreading/ patent, obovate, crisped leaves. Leaf base plain. Leaf apex obtuse and mucronate. Leaf margin serrate. Absence of alar cells. Costa below apex. Capsule pendulous, large, with hypophysis, conical operculum, large peristome teeth.

Habitat: on decayed log/litter
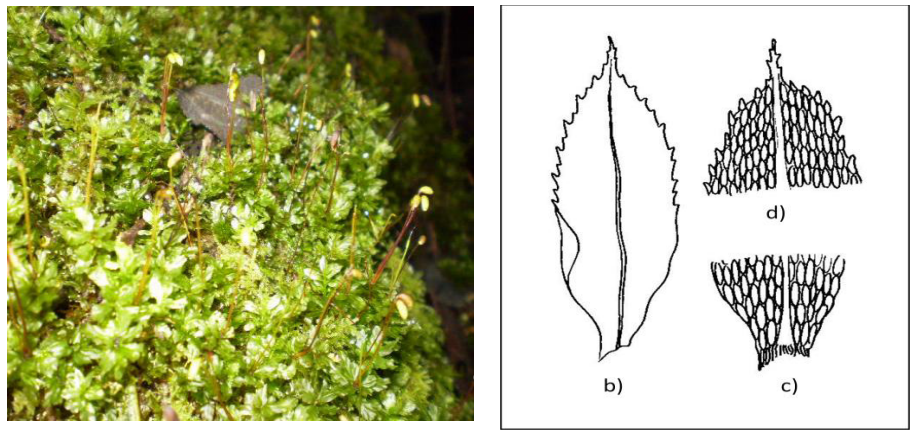

Fig. 3. FAMILY BRYACEAE

Bryum sp.

(see next page for legend) 

a) Plant habit
b) Leaf
c) Basal leaf cells
d) Apical leaf cells; drawn from x 100

Description. Plants in tufts. Stems erect radiculose below, often subfloral innovations. Upper leaves usually larger, lower leaves small, lanceolate; costa ending in or near apex; cells linear or rhomboidal , thin-walled, smooth, often narrower in several rows at margins. Seta elongate; capsule inclined or pendulous, rarely erect, clavate or pyriform with a distinct, tapering neck; peristome double. 16 lanceolate teeth at outer, inner rudimentary or composed of 16 keeled segments alternating with teeth from a high basal membrane; lid short, conical.

Habitat: on soil, decayed rock surface
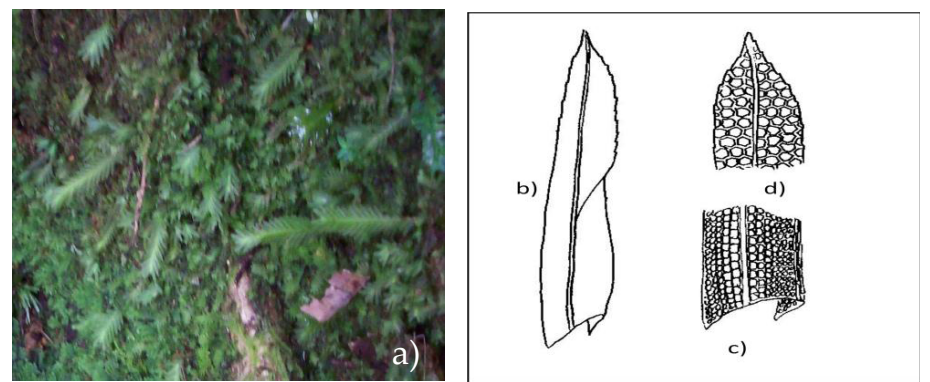

Fig. 4. FAMILY FISSIDENTACEAE

Fissidens nobilis Griff.
a) Plant habitat
b) Leaf
c) Basal leaf cells
d) Apical leaf cells; drawn from x 100

Description: Plants small to medium, quite robust, flattened leaves in one plane. Dark green when fresh and pale green when dry. Leaves spreading/ patent, oblong-lanceolate, with axillary leaves. Leaf base cordate. Leaf apex rounded obtuse. Leaf margin entire. Absence of alar cells. Costa up to apex, very distinct.

Habitat: on soil, rock surface 

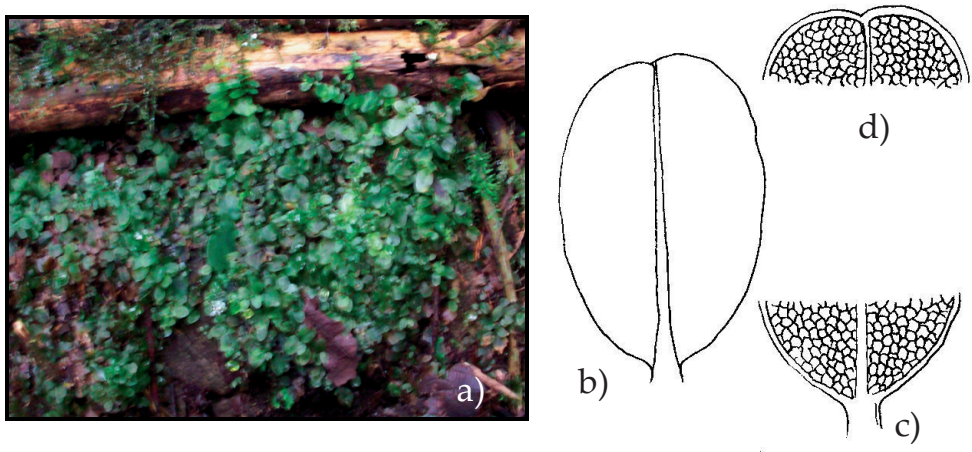

Fig. 5. FAMILY MNIACEAE

Plagiomnium sp.
a) Plant habitat
b) Leaf
c) Basal leaf cells
d) Apical leaf cells; drawn from x 100

Description: Plants medium, slender, forming tufts or cushion. Dark green when fresh and pale yellow-green when dry. Leaves spreading/ patent, lingulate. Leaf base plain. Leaf apex apiculate, mucronate. Leaf margin dentate. Absence of alar cells. Costa up to apex, thick. Capsule inclined to pendulous, ovoid, quite long, several peristome teeth.

Habitat: on decayed log; soil
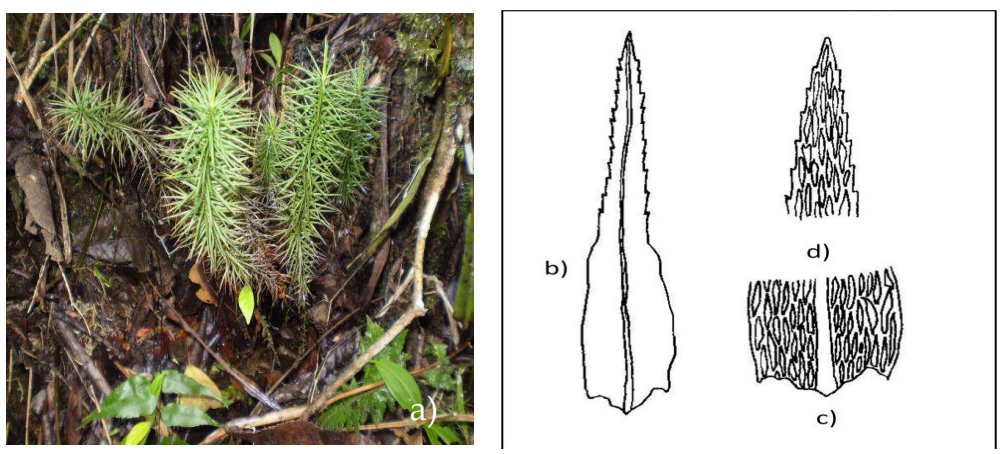

Fig. 6. FAMILY POLYTRICHACEAE

Dawsonia superba Grev.

(see next page for legend) 
a) Plant habitat

b) Leaf

c) Basal leaf cells

d) Apical leaf cells; drawn from x 100

Description: Plants large, robust, erect stem. Dark green when fresh and dark brownish red with green when dry. Leaves spreading/ patent, linear, involute base. Leaf base plain. Leaf apex acute, mucronate. Leaf margin dentate. Absence of alar cells. Costa from base up apex.

\section{Habitat: on soil}
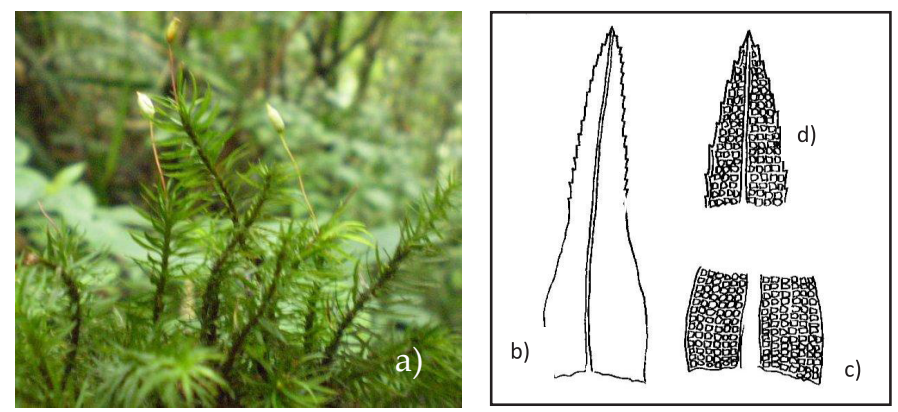

Fig. 7. FAMILY POLYTRICHACEAE

Pogonatum macrophyllum (Dozy \&Molk.) Lindb.

e) Plant habitat

f) Leaf

g) Basal leaf cells

h) Apical leaf cells; drawn from x 100

Description: Plants large, robust, falcate, stems erect. Dark green when fresh and green when dry. Leaves spreading/ patent, linear lanceolate, involute from base up to the apex. Leaf base plain. Leaf apex acute. Leaf margin dentate. Absence of alar cells. Costa up to apex, distinct. Capsule acrocarpous, erect, with hypophysis, several peristome teeth.

Habitat: on soil 

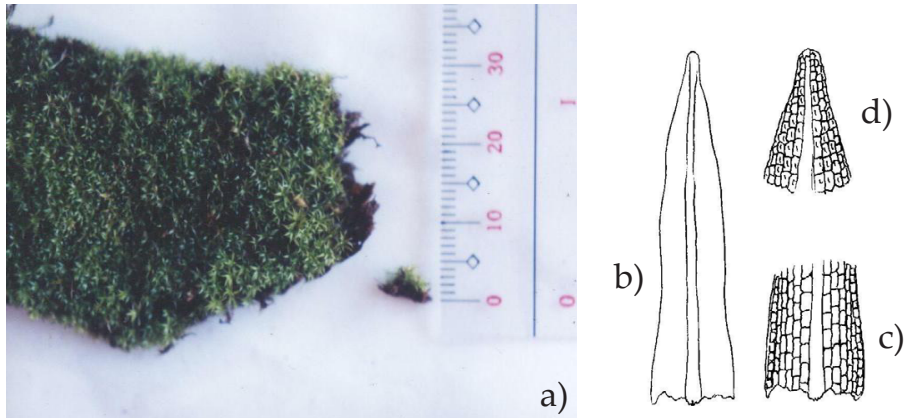

Fig. 8. FAMILY POTTIACEAE

Barbula sp.

a) Plant habit

b) Leaf

c) Basal leaf cells

d) Apical leaf cells; drawn from x 100

Description: Plants small, robust, erect, forming tuft or cushion. Green when fresh and yellow green to brown when dry. Leaves spreading/ patent, lanceolate,involute. Leaf base cordate. Leaf apex setaceous. Leaf margin entire. Absence of alar cells. Costa up to apex, distinct.

Habitat: on tree trunk
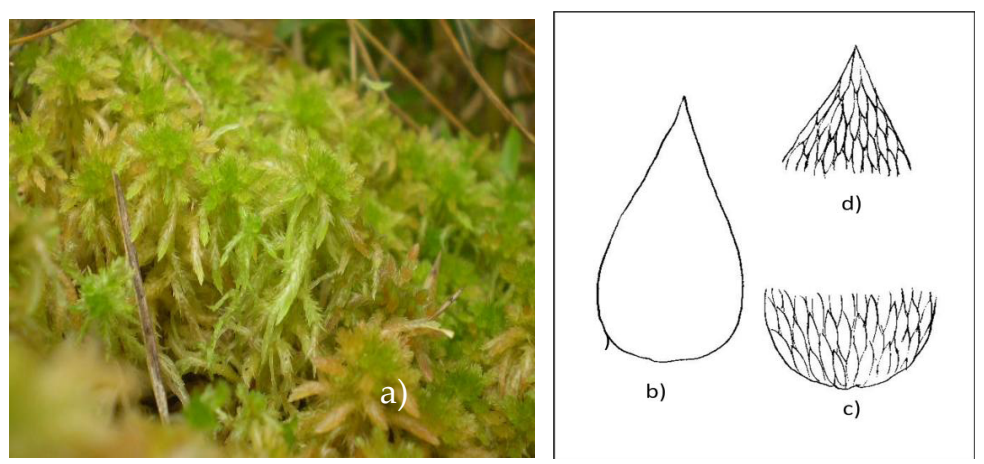

Fig. 9. FAMILY SPHAGNACAE

Sphagnum sericeum C. Mull.

a) Plant habitat 
b) Leaf

c) Basal leaf cells

d) Apical leaf cells; drawn from x 100

Description: Plants medium to large, slender. Yellow-green when fresh and whitish green when dry. Leaves spreading/ patent, oblonglanceolate, slightly involute in two (2) sides. Leaf base plain. Leaf apex acute. Leaf margin entire. Absence of alar cells. Ecostate.

Habitat: on decayed log/ thick litter
A. Liverworts
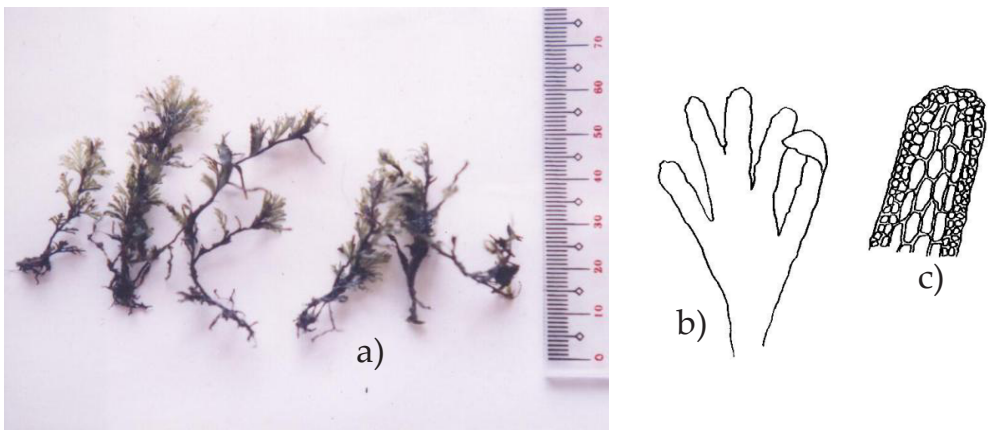

Fig. 10. FAMILY ANEURACEAE

Riccardia sp.
a) Plant habit
b) Leaf
c) Leaf cells

Description: Thalloid liverworts. Green when fresh and blackish brown when dry. Pinnately branching. Forkingstem leaf.

Habitat: on soil; on decayed log 

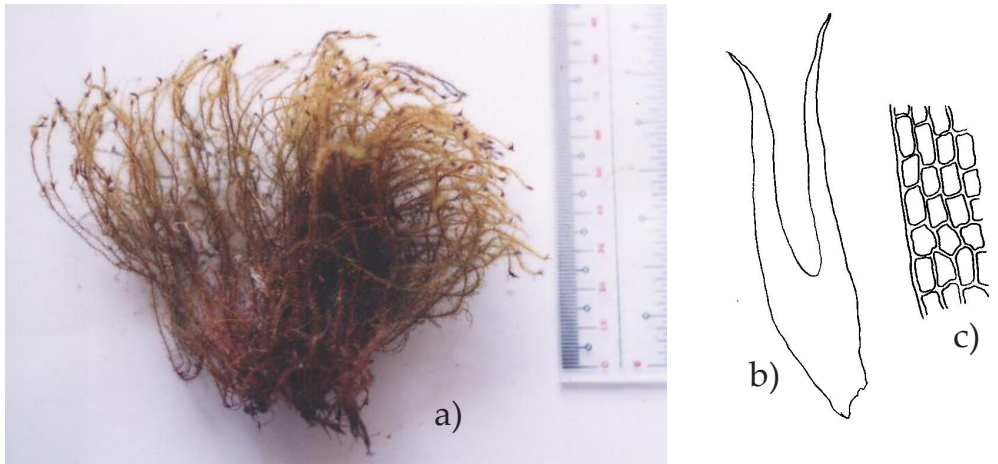

c)

Fig. 11. FAMILY HERBERTACEAE

Herbertus $s p$.

a) Plant habit

b) Leaf

c) Leaf cells

Description: Leafy liverworts. Reddish green when fresh and blackish brown when dry, branching. Leaves spreading/ patent at the upper end of the stem and imbricate at the lower part. Incubous. Lateral leaves stout, bifid;leaf base decurrent and broad; leaf margin entire. Absence of ventral/ underleaves. Presence of vitta.

Habitat: on decayed log
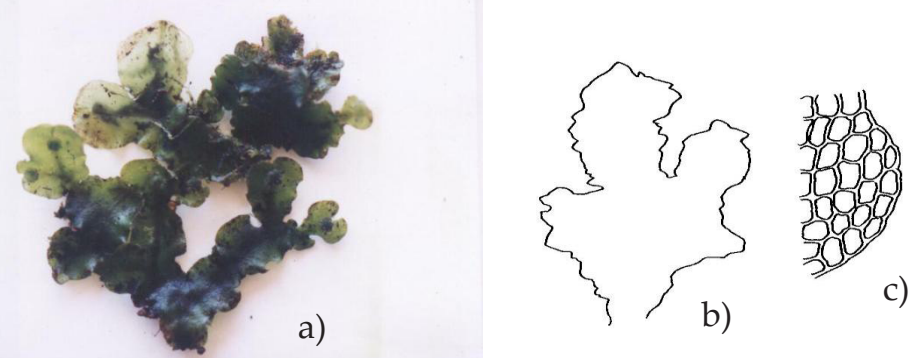

Fig. 12. FAMILY MARCHANTIACEAE

Dumortiera sp.
a) Plant habit
c) Leaf cells
b) Leaf 
Description: Thalloid liverwort. Green when fresh and dry. Leaves in many lobes, leaf base plain; leaf margin entire; leaf apex round. Presence of "vitta".

Habitat: on decayed $\log$, soil
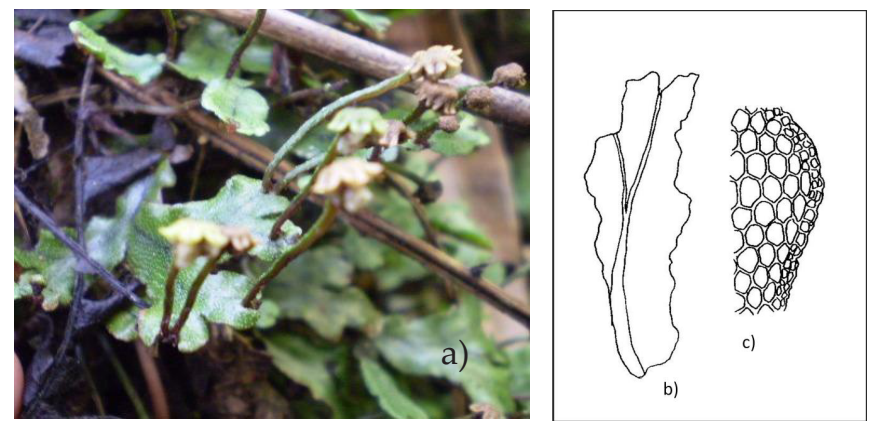

Fig. 13. FAMILY MARCHANTIACEAE

Marchantia polymorpha
a)Plant habit
b)Leaf
c)Leaf cells

Description: Thalloid liverwort. Green when fresh and brownish green when dry, dichotomously branched, thick. Leaves in many lobes; leaf base plain; leaf margin entire; leaf apex round. Presence of antheridia arising on stalked disc-headed branched, the antheridophores. Presence of "costa", thick.

Habitat: on soil 

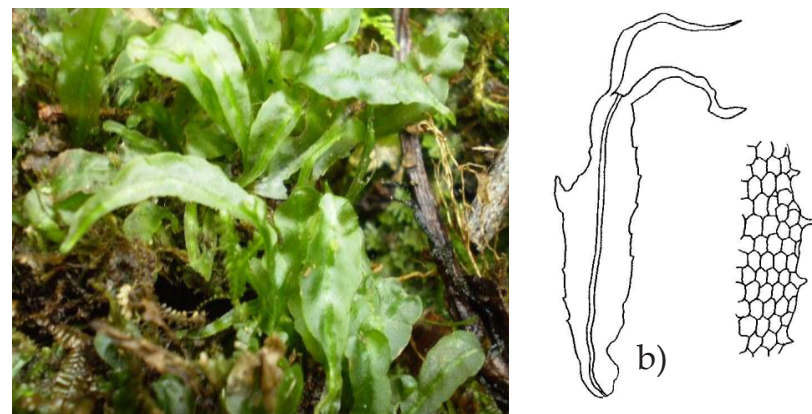

c)

Fig. 14. FAMILY PALLAVICINIACEAE

Pallavicinia sp.
a) Plant habit
b) Leaf
c) Leaf cells

Description: Thalloid liverworts. Green when fresh and greenish brown when dry, dichotomous branched. Leaves in many lobes; leaf base plain; leaf margin dentate; leaf apex round. Presence of "vitta". Capsule pleurocarpous, oblong, very short seta, stout.

Habitat: on decayed log; soil
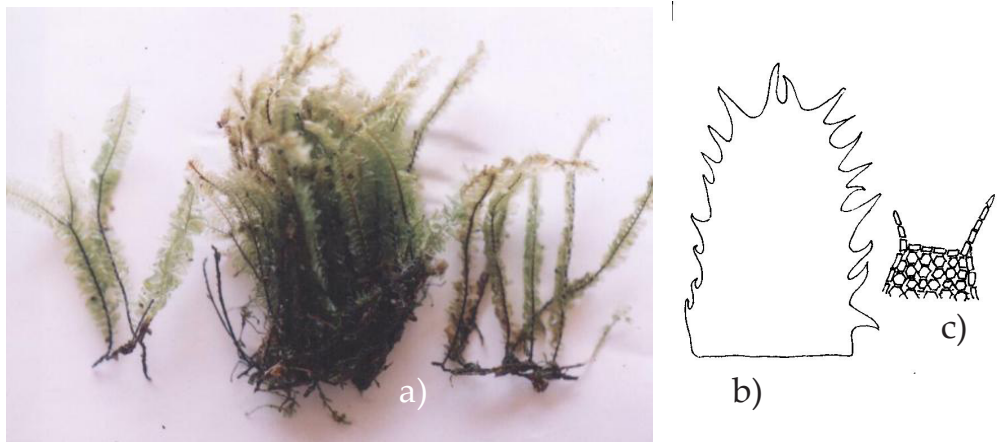

b)

Fig. 15. FAMILY PLAGIOCHILACEAE Plagiochila sp.
a) Plant habit
b) Leaf
c) Leaf cells 
Description: Leafy liverworts. Green when fresh and brown when dry, branching. Succubous. Lateral leaves cultriform, involute base, leaf base nearly decurrent; leaf margin dentate, not so observable; leaf apex obtuse. Ventral/underleaves spathulate, smaller.

\section{Habitat: on decayed $\log$}

\section{Table 1. Medicinal bryophytes, their morphology and habitat preference}

\begin{tabular}{|c|c|c|}
\hline Bryophytes Species & Morphology features & $\begin{array}{l}\text { Habitat } \\
\text { Preference }\end{array}$ \\
\hline
\end{tabular}

\section{MOSSES}

1. Bartramiaceae

Philonotis sp.

\section{Bryaceae}

\section{Rhodobryum giganteum}

(Schwaegr.)

Par.

Bryum sp.
Plants slender, erect. Green when fresh and whitish yellow green when dry. Leaves spreading/ patent, oblong ovate, involute leaves. Leaf base plain. Leaf apex setaceous. Leaf margin entire. Absence of alar cells. Costa up to apex. Capsule erect, acrocarpous, ovoid, absence of operculum, long seta.

Plants large size, forming tufts. Dark green when fresh and green when dry. Leaves spreading/ patent, obovate, crisped leaves. Leaf base plain. Leaf apex obtuse, mucronate. Leaf margin serrate. Absence of alar cells. Costa below apex. Capsule pendulous, large, with hypophysis, conical operculum, large peristome teeth.

Plants in tufts. Stems erect radiculose below, often subfloral innovations. Upper leaves usually larger, lower leaves small, lanceolate; costa ending in or near apex; cells linear or rhomboidal, thin-walled, smooth, often narrower in several rows at margins. Seta elongate; capsule inclined or pendulous, rarely erect, clavate or pyriform with a distinct, tapering neck; peristome double. on tree trunk

on decayed $\log /$ litter

on soil; decayed rock surface 
3. Fissidentaceae

Fissidens nobilis Griff. Plants small to medium, quite robust, flattened leaves in one plane. Dark on rock green when fresh and pale green when surface; soil dry. Leaves spreading/ patent, oblonglanceolate, with axillary leaves. Leaf base cordate. Leaf apex rounded obtuse. Leaf margin entire. Absence of alar cells. Costa up to apex, very distinct.

\section{Mniaceae}

Plagiomnium sp.

Plants medium, slender, forming tufts or on decayed cushion. Dark green when fresh and pale yellow-green when dry. Leaves spreading/ patent, lingulate. Leaf base plain. Leaf apex apiculate, mucronate. Leaf margin dentate. Absence of alar cells. Costa up to apex, thick. Capsule inclined to pendulous, ovoid, quite long, several peristome teeth.

Mnium sp.

Medium-sized. Leaves broad, abruptly pointed, firm, leathery; crispate when dry, on soil oblong, short-pointed, usually bordered; serrate margins. Stems erect with upper leaves crowded in rosulate tufts, sterile branches often prostate and equally foliate. Seta terminal , elongate; capsule horizontal or erect; peristome double; calyptra small, cucullate.

\section{Polytricaceae}

Dawsonia superba Grev.

Plants large, robust, erect stem. Dark green

on soil when fresh and dark brownish red with green when dry. Leaves spreading/ patent, linear, involute base. Leaf base plain. Leaf apex acute, mucronate. Leaf margin dentate. Absence of alar cells. Costa from base up to apex.

Pogonatum macrophyllum Plants large, robust, falcate, stems erect. Dozy \& Molk. Dark green when fresh and green when dry. Leaves spreading/ patent, linear lanceolate, involute from base up to the apex. Leaf base plain. Leaf apex acute. Leaf margin dentate. Absence of alar cells. Costa up to apex, distinct. Capsule acrocarpous, erect, with hypophysis, several peristome teeth.

on soil 
Polytrichum sp.
Medium to robust plants. Erect, simple or sparingly branched stems. Leaves narrow, rigid, blade usually well differentiate from sheathing base; costa strong, percurrent to excurrent, often toothed on back above, usually broader on ventral surface and covered with thin, parallel, longitudinal lamellae. Seta elongate; capsule erect or inclined.

6. Pottiaceae

Barbula sp.

Plants small, robust, erect, forming tuft or cushion. Green when fresh and yellow green to brown when dry. Leaves spreading/ patent, lanceolate, involute. Leaf base cordate. Leaf apex setaceous. Leaf margin entire. Absence of alar cells. Costa up to apex, distinct.

7. Sphagnaceae

Sphagnum sericeum C. Mull.
Plants medium to large, slender. Yellowgreen when fresh and whitish green when dry. Leaves spreading/ patent, oblonglanceolate, slightly involute in two (2) sides. Leaf base plain. Leaf apex acute. Leaf margin entire. Absence of alar cells. Ecostate. on rock; soil

On tree trunk

on decayed $\log$ /thick litter

\section{LIVERWORTS}

1. Aneuraceae

Riccardia sp.

Thalloid liverworts. Green when fresh and blackish brown when dry. Pinnately branching. Forking stem leaf.

on soil; decayed log

2. Herbertaceae

Herbertus sp. Leafy liverworts. Reddish green when fresh on decayed log and blackish brown when dry, branching. Leaves spreading/ patent at the upper end of the stem and imbricate at the lower part. Incubous. Lateral leaves stout, bifid;leaf base decurrent and broad; leaf margin entire. Absence of ventral/ underleaves. Presence of vitta. 
3. Marchanticeae

Dumortiera

sp.

Marchantia sp.

4. Pallaviciniaceae

Pallavicinia sp.

5. Plagiochilaceae

Plagiochila sp.
Thalloid liverwort. Green when fresh and dry. Leaves in many lobes, leaf base plain; leaf margin entire; leaf apex round. Presence of "vitta".

Thalloid liverwort. Green when fresh and brownish green when dry, dichotomously branched, thick. Leaves in many lobes; leaf base plain; leaf margin entire; leaf apex round. Presence of antheridia arising on stalked disc-headed branched, the antheridophores. Presence of "costa", thick.

Thalloid liverworts. Green when fresh and greenish brown when dry, branched. Leaves in many lobes; leaf base plain; leaf margin entire; leaf apex round. Presence of "vita".

Leafy liverworts. Green when fresh and brown when dry. Incubous. Lateral leaves cultriform-lingulate, leaf base plain; leaf margin entire; leaf apex toothed in one side, entire in the other side. Absence of ventral/ underleaves. on decayed

$\log$; soil

on soil

on decayed

log; soil

on decayed log

\section{Table 2. List of medicinal bryophytes, their distribution, and medical uses}

\begin{tabular}{llll}
\hline $\begin{array}{l}\text { Medicinal Bryophytes } \\
\text { Species }\end{array}$ & $\begin{array}{l}\text { Place of } \\
\text { Distribu- } \\
\text { tion }\end{array}$ & Medical Uses & Active Components \\
\hline
\end{tabular}

\section{MOSSES}

1. Bartramiaceae

Philonotis sp. Kit

Heal burns Triterpenoidalsaponins

For adenopharyngitis, antipyretic and antidotal (Asakawa, 2007) 


\section{Bryaceae}

Rhodobryum giganteum (Schwaegr.) Par.

Bryum sp.

Kit

3. Fissidentaceae

Fissidens nobilis Griff.

\section{Mniaceae}

Plagiomnium sp.

Mnium sp.

\section{Polytrichaceae}

Dawsonia superba Grev.

Pogonatum macrophyllum Dozy \& Molk.

Polytrichum sp.
Kit, Kal

For cardiovascular problem and nervous prostration; to cure angina; anti-hypoxia Antipyretic, diuretic and antihypertensive (Asakawa 2007)

Healing wounds, burns and bruises; cure fungal infections (Beike et al. 2010)

Kit, Kal

Diuretics and hair growth stimulation tonics

As antibacterial agent for swollen throats and other symptoms of bacterial infection

$\begin{array}{ll}\text { Kit, Kal } & \begin{array}{l}\text { For infections and } \\ \text { swellings }\end{array} \\ \text { Kit } & \begin{array}{l}\text { Poultice to reduce pain } \\ \text { of burns, bruises and } \\ \text { wounds }\end{array}\end{array}$

Kit, Kal Diuretics and hair growth stimulation; for treating colds

Kit, Kal

To reduce inflammation and fever, as detergent diuretic, laxative and hemostatic agent

Kit, Kal Healing burns, bruises and wounds; used against fever and inflammation (Beike et al. 2010) Antipyretic and antidotal; for hemostasis, cuts, bleeding from gingivae
P-hydroxycinnamic

Acid

7-8 dihydroxycoumarin

Triterpenoidalsaponins

Triterpenoidalsaponins 


\section{Pottiaceae}

Barbula sp.

Boiled as tea for treat-

ing colds; for fever and

body aches (Sturtevant 1954)

\section{Sphagnaceae}

Sphagnum sericeum C.

Mull.

\section{LIVERWORTS}

1. Aneuraceae

Riccardia sp.

Kit, Kal

Exhibits anti-leukemic activity

Riccardins A (57) and B (58), Sacullatal (15)*

2. Herbertaceae

Herbertus sp.

Kit, Ka

As antiseptics, antidiarrheal agents, expectorants, astringents

1. Marchanticeae

Dumortiera hirsute

Marchantia polymorpha

Kit, Kal

Kit, Kal

As source for antibiotics

As diuretics, for liver ailments, insect bites, boils and abscesses; treat pulmonary tuberculosis; with growthinhibiting substances; used to cure cuts, fractures, poisonous snake bites, burns, scalds and open wounds; for cardiovascular disease (Beike et al. 2010)
Marchantin A (61);

MB-G (35a)

Marchantin D (65) and E (66)*

1. Pallaviciniaceae

Pallavicinia sp. Kit, Kal

Extracts for antimicro- Sacullatal (15)* bial activity 
2. Plagiochilaceae

Plagiochilasp. Kit, Kal Exhibits anti-leukemic Bicyclohumulenone (3), activity/ anti-microbial Plagiochiline A (13), activity

Plagiochilide (81),

Plagiochilal B (19); Menthanemonoterpenoids*

\section{Table 3. Number of families, genera and medicinal species of bryophytes in the two Natural Parks, Bukidnon}

\begin{tabular}{|c|c|c|c|c|c|c|c|c|c|}
\hline \multirow{2}{*}{ Study Area } & \multicolumn{2}{|c|}{ Family } & \multirow[t]{2}{*}{ Total } & \multicolumn{2}{|c|}{ Genera } & \multirow[t]{2}{*}{ Total } & \multicolumn{2}{|l|}{ Species } & \multirow[t]{2}{*}{ Total } \\
\hline & $\begin{array}{l}\text { Moss- } \\
\text { es }\end{array}$ & $\begin{array}{l}\text { Liver- } \\
\text { worts }\end{array}$ & & $\begin{array}{l}\text { Moss- } \\
\text { es }\end{array}$ & $\begin{array}{l}\text { Liver- } \\
\text { worts }\end{array}$ & & Mosses & $\begin{array}{l}\text { Liver- } \\
\text { worts }\end{array}$ & \\
\hline Mt. Kitanglad & 7 & 5 & 12 & 11 & 6 & 17 & 11 & 6 & 17 \\
\hline Mt. Kalatungan & 6 & 5 & 11 & 8 & 6 & 14 & 8 & 6 & 14 \\
\hline
\end{tabular}

Table 4. Medical bryophytes and ethnobotanical practices and other uses

\begin{tabular}{|c|c|}
\hline Bryophytes Species & Ethnobotanical Practices/Other Uses \\
\hline MOSSES & \\
\hline 1. Bartramiaceae & \\
\hline Philonotis sp. & $\begin{array}{l}\text { Poultice as padding under splints to set broken bones } \\
\text { As chinking in temporary summer homes for shepherds use } \\
\text { (G.B. Pant and S.D. Tewari 1989) } \\
\text { Crushed unto a kind of paste and applied as a poultice by } \\
\text { the Chinese and the Native Americans (D. K. Saxena \& } \\
\text { Harinder 2004) }\end{array}$ \\
\hline
\end{tabular}




\begin{tabular}{|c|c|}
\hline 2. Bryaceae & \\
\hline $\begin{array}{l}\text { Rhodobryum giganteum } \\
\text { (Schwaegr.) Par. }\end{array}$ & $\begin{array}{l}\text { Extract to cure angina }(\mathrm{Wu}, 1977) \\
\text { Prevent soil erosion of the slope of hills because the netted } \\
\text { and wobbed protonema cover the exposed substrates (D. K. } \\
\text { Saxena \& Harinder 2004) } \\
\text { Can increase aorta blood transit by up to } 30 \% \text { in animals (D. } \\
\text { K. Saxena \& Harinder 2004) }\end{array}$ \\
\hline Bryum sp. & $\begin{array}{l}\text { Poultice as padding under splints to set broken bones } \\
\text { External applications for fever and body aches (Sturtevant } \\
\text { 1954) }\end{array}$ \\
\hline 3. Fissidentaceae & \\
\hline Fissidens nobilis Griff. & $\begin{array}{l}\text { Burned to put on their heads to encourage hair growth } \\
\text { (Harris 2002) }\end{array}$ \\
\hline 4. Mniaceae & \\
\hline Plagiomnium sp. & $\begin{array}{l}\text { Poultice of crushed "leaves" used for blood blisters, breast } \\
\text { abscesses }\end{array}$ \\
\hline Mnium sp. & Poultice as padding under splints to set broken bones \\
\hline 5. Polytrichaceae & \\
\hline Dawsonia superba Grev. & \\
\hline $\begin{array}{l}\text { Pogonatum macrophyllum } \\
\text { Dozy \& Molk. }\end{array}$ & As inhibitors of soil erosion (D. K. Saxena \& Harinder 2004) \\
\hline Polytrichum sp. & $\begin{array}{l}\text { Boiled as tea for treating the cold (Gulabani } 1974 \text { in Beike et } \\
\text { al 2010) } \\
\text { Women in labor chewed the moss to speed up labor process } \\
\text { (Turner et al. 1983) } \\
\text { Used as nautical ropes (J.H. Dickson 1973) } \\
\text { Used to line as carpets during winter hibernation sites; } \\
\text { when animals bury their food in wet forest they serve } \\
\text { as line mats (J. Hyvonen 1990) } \\
\text { For bedding material } \\
\text { Used in landscape tray, an alternative horticultural art of } \\
\text { Japan (D. K. Saxena \& Harinder 2004) }\end{array}$ \\
\hline
\end{tabular}




\begin{tabular}{|c|c|}
\hline 6. Pottiaceae & \\
\hline Barbula sp. & $\begin{array}{l}\text { As antirheumatic (external) febrifuge; external applications } \\
\text { for fever and body aches (Sturtevant 1954) }\end{array}$ \\
\hline 7. Sphagnaceae & \\
\hline $\begin{array}{l}\text { Sphagnum sericeum } \mathrm{C} \text {. } \\
\text { Mull. }\end{array}$ & $\begin{array}{l}\text { Brewed like a tea as heat tonic (Shevock 1997) } \\
\text { Used for construction when dried and blended with } \\
\text { phenolic resins and pressed into heating mold (Ruel et al } \\
\text { 1977) } \\
\text { As stuffed between timbers of houses to deaden sound (J. } \\
\text { W. Thieret 1954) } \\
\text { Used by Russians to insulate homes and refrigerators (M.A. } \\
\text { Sukhanov 1972; M. Ruel et al. 1977) } \\
\text { Used to mixed with light concrete and hydraulically pressed } \\
\text { with Portland cement and water as peat crete } \\
\text { Used to line hiking boots, where it absorbs moisture and } \\
\text { odor; and as lining diaper (L. Hedenas 1991; J.H. Bland } \\
\text { 1971) } \\
\text { Used as sanitary napkins (L.M. Johnson Gottesfeld and D. } \\
\text { H. Vitt, 1996) } \\
\text { As fillers between wooden posts of walls and shingles of } \\
\text { roofes (B.C. Tan 2003) } \\
\text { As chinking between timbers in homes (J. Glime 2007) } \\
\text { Provide suitable substrates for the biological fixation of } \\
\text { nitrogen in association with cyanobacteria (D. K. Saxena \& } \\
\text { Harinder, 2004) } \\
\text { Used in air layering, a method of propagating plants (D. K. } \\
\text { Saxena \& Harinder 2004) } \\
\text { Mixed with the soil or spread over the ground as a mulch } \\
\text { (D. K. Saxena \& Harinder 2004) } \\
\text { Helps to maintain moisture and prevent growth of weeds } \\
\text { (D. K. Saxena \& Harinder 2004) }\end{array}$ \\
\hline
\end{tabular}




\begin{tabular}{|c|c|}
\hline & $\begin{array}{l}\text { Used as an effective filtering and absorption agent for the } \\
\text { treatment of waste water and effluent of factories with acid } \\
\text { and toxic discharge containing heavy etals }(\mathrm{Ag}, \mathrm{Cu}, \mathrm{Cd} \text {, } \\
\mathrm{Hg}, \mathrm{Fe}, \mathrm{Sb} \text { and } \mathrm{Pb} \text { ) and organic substances such as oils, } \\
\text { detergents, dyes and micro-organisms (D. K. Saxena \& } \\
\text { Harinder 2004) } \\
\text { An excellent material for shipment of plants of fresh } \\
\text { vegetables and flowers; for hydrophysics gardening, and } \\
\text { for storage of roots and bulbs; used in the manufacturing of } \\
\text { insulator sheets for houses (D. K. Saxena \& Harinder 2004) } \\
\text { Mixed with worl has ben used to prepare a cheap cloth (D. } \\
\text { K. Saxena \& Harinder 2004) } \\
\text { Is wet often used by nurserymen in India for sending or } \\
\text { supplying live plants (D. K. Saxena \& Harinder 2004) } \\
\text { Used as Sphagnum pads for dressing wounds (D. K. Saxena } \\
\text { \& Harinder 2004) }\end{array}$ \\
\hline LIVERWORTS & \\
\hline 1. Aneuraceae & \\
\hline Riccardia sp. & \\
\hline 2. Herbertaceae & \\
\hline Herbertus sp. & $\begin{array}{l}\text { As chinking in temporary summer homes for shepherds use } \\
\text { (G. B. Pant and S.D. Tewari 1989) }\end{array}$ \\
\hline 3. Marchanticeae & \\
\hline Dumortiera sp. & \\
\hline Marchantia sp. & For boils and abscesses (D. K. Saxena \& Harinder 2004) \\
\hline 4. Pallaviciniaceae & \\
\hline Pallavicinia sp. & \\
\hline 5. Plagiochilaceae & \\
\hline Plagiochila sp. & $\begin{array}{l}\text { As chinking in temporary summer homes for shepherds use } \\
\text { (G. B. Pant and S.D, Tewari 1989) } \\
\text { Used as perfumes or as perfume components (Asakawa } \\
\text { 2007) } \\
\text { Non-ionized organic acids and polyphenolic compounds (D. } \\
\text { K. Saxena \& Harinder 2004) }\end{array}$ \\
\hline
\end{tabular}


Table 5. List of Non-medicinal bryophytes covered in the study and their economic uses

\begin{tabular}{|l|l|}
\hline Bryophytes Species & Economic Uses \\
\hline $\begin{array}{l}\text { Mosses } \\
\text { 1. Leucobryaceae }\end{array}$ & $\begin{array}{l}\text { For decorative arrangements such as floral } \\
\text { arrangements and as cushions }\end{array}$ \\
\hline Leucobryum glaucum & $\begin{array}{l}\text { As chinking in temporary summer homes for } \\
\text { shepherds use (G. B. Pant and S.D. Tewari } \\
1989)\end{array}$ \\
\hline 2. Meteoriaceae & $\begin{array}{l}\text { As insect repellant and resistance to rot (G. B. } \\
\text { Pant and S.D. Tewari 1989) }\end{array}$ \\
\hline Floribundaria sp. & $\begin{array}{l}\text { Used to seal seams and cracks of boats and } \\
\text { canoes (G. B. Pant and S.D. Tewari 1990) }\end{array}$ \\
\hline 3. Neckeraceae & $\begin{array}{l}\text { Used for packing apples and planes in } \\
\text { Western Himalayas (D. K. Saxena \& } \\
\text { Harinder 2004) }\end{array}$ \\
\hline Neckera sp. & \begin{tabular}{l} 
As chinking in temporary summer homes for \\
shepherds use (G. B. Pant and S.D. Tewari \\
1989) \\
Used as carpets for nativity scenes in USA \\
As insect repellant and resistance to rot (G. B. \\
Pant and S.D. Tewari 1989) \\
Used for packing apples and planes in \\
Western Himalayas (D. K. Saxena \& \\
\hline H. Thuidiaceae
\end{tabular} \\
\hline Thuidium sp. & \begin{tabular}{l} 
Harinder 2004) \\
\hline
\end{tabular} \\
\hline
\end{tabular}




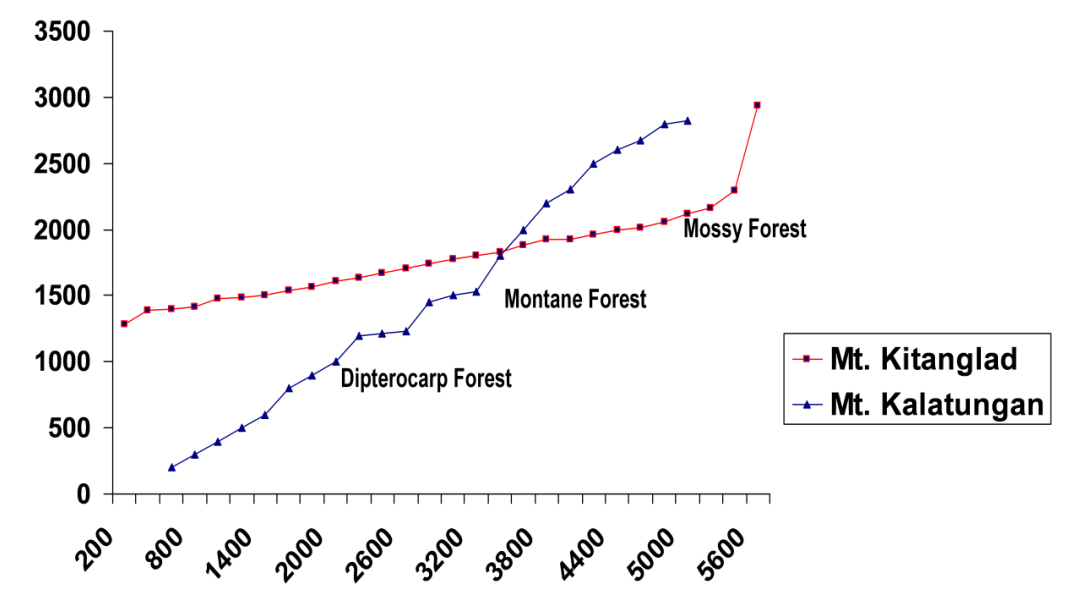

Fig. 1. Transect map of the two mountain sites.

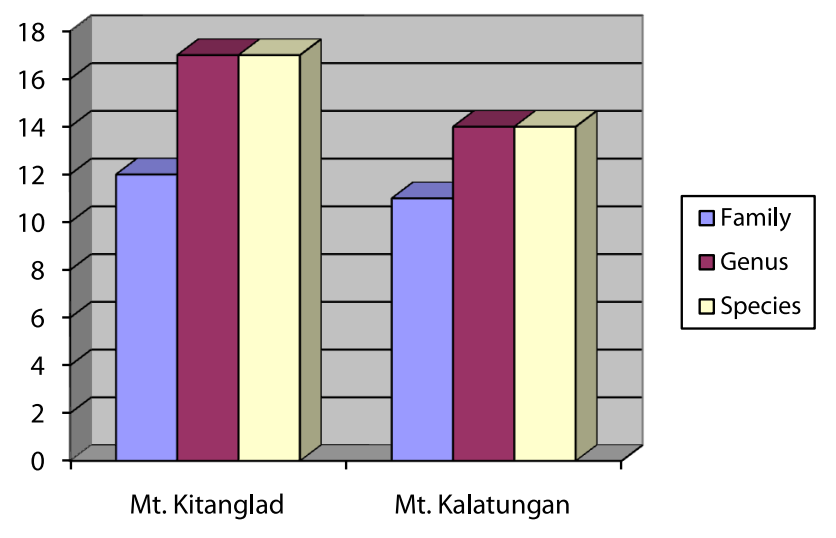

Fig 2. Graph on species richness of medicinal bryophytes to compare the two study are. 
B. Species Richness and Composition

A total of eleven (11) species of mosses and six (6) species of liverworts are in Mt. Kitanglad while eight (8) species of mosses and six (6) species of liverworts are in Mt. Kalatungan. These species were all reported with potential medicinal properties. The study identified seven (7) families, eleven (11) genera and eleven (11) species of mosses while five (5) families, six (6) genera and six (6) species of liverworts (Table 3 and Fig. 2). The moss species that were identified with medicinal properties include Sphagnum sericium, Polytrichum sp., Rhodobryum giganteum, Fissidens nobilis, Bryum sp., Mnium sp., Dawsonia superba, Philonotis sp., Pogonatum macrophyllum, Barbula sp. and Plagiomnium sp.The liverworts species include Marchantia polymorpha, Pallaviciniasp., Herbertus sp., Riccardia sp., Dumortiera hirsuta and Plagiochila sp. (Table 2).

\section{CONCLUSIONS}

\section{The following conclusions were drawn:}

1. A total of 17 species of medicinal bryophytes were collected in Mt. Kitanglad. Eleven (11) are mosses belonging to 7 families and 11 genera. Six (6) liverworts belonging to 5 families in 6 genera while Mt. Kalatungan exhibited 14 species of medicinal bryophytes. Of these, 8 are mosses belonging to 6 families in 8 genera and 6 liverworts belonging to 5 families in 6 genera. In terms of species richness, Mt. Kitanglad shows more diverse than Mt. Kalatungan.

2. The moss species were identified with medicinal properties include Sphagnum sericium, Polytrichum sp., Rhodobryum giganteum, Fissidens nobilis, Bryum sp., Mnium sp., Dawsonia superba, Philonotis sp., Pogonatum macrophyllum, Barbula sp. and Plagiomnium sp. The liverworts species include Marchantia polymorpha, Pallavicinia sp., Herbertus sp., Riccardia sp., Dumortiera hirsuta and Plagiochila sp.

3. Based on literature review, the mosses and liverworts were found to possess certain biological activity. As such the presences 
of active components have been widely used as medicinal plants. Treatment include antimicrobial, antifungal activity, antidotal , diuretics, antipyretic, antileukemic, antitumor antihypertensive, antidiarrheal , anticancer, cardiovascular diseases, inflammation, fever, infections, wounds and skin diseases and healing effects. Likewise, other ethnic and valuable uses were noted.

4. Morphological diversity existed among species of bryophytes with medical properties. Each are distributed in various habitats with habitat preferences such as in soil, decayed logs, litters, tree trunks, rocks surfaces and decayed and non-decayed rocks.

\section{RECOMMENDATIONS}

It is noteworthy that species of bryophytes have placed taxonomic and ecological importance in the natural forest parks. More importantly, the literature review is an important tool to bryologists, taxonomists, scientists, biologists, students, and educators in providing the knowledge-based information on the potential medical uses. This would guide biotechnologists to investigate further research and explore bryotechnology as new avenues for applied research. With this, scientific investigation should be conducted in different forest types and reassess the active compounds present in the potential medicinal species of bryophytes through biological activity.

\section{LITERATURE CITED}

Asakawa, Y.

2007 Biologically active compounds from bryophytes. Pure Applied Chemistry. 79: 557-580.

Asakawa, Y.

2008 Liverworts-potential source of medicinal compounds. Current Pharmaceutical Design.Bentham Science Publishers. 
Azuelo, A. G.

2005 Bryophyte flora of Mt. Malindang, Misamis Occidental , Philippines, Dissertation. Central Mindanao University.

Beike, A.K., E. Decker, F. Wolfgang. D. Lang, M. Verulit- Scheebaum, A. Zimmer, And R. Reski.

2010 Applied Bryology-Bryotechnology Tropical Bryology. 31: 2232.

Cox, C. J., B. Goffinet, N. J. Wickett, S. B. Boles And A.J. Shaw.

2010 Moss diversity: A molecular phylogenetic analysis of genera. Phytotaxa : 175-195.

Gignac, L. D.

2001 New frontiers in bryology and lichenology: Bryophytes as indicators of climate change. The Bryologist 104: 410-420.

Glime, J.M.

1991 Economic and ethnic uses of bryophytes. General Article.

Gulaban, A.

1974 Bryophytes as economic plants. Botanica 14: 73-75.

Hallingback, T. and N. Hodgetts.

2000 Status survey and conservation action plan for bryophytes: mosses, liverworts and hornworts. IUCN/SSC Bryophyte Specialist Group. IUCN, Gland, Switzerland and Cambridge, U.K. 106 pp.

Hallingback, T. And B.C. Tan.

2010 Past and present activities and future strategy of bryophytes conservation. Phytotaxa 9: 266-274.

Harris, E.

2002 An examination of phylogenetic characters in mosses: Examples from Fissidens Hedw. (Fissidentaceae: Musci). Presentation and abstract presented at the annual meeting of the American Bryological and Lichenological Society, 26-27 July 2002, Storrs, 
CN, USA.

Lubos, L. C.

2000 Taxonomy, species richness and distribution of mosses in selected mountains in Mindanao. Dissertation. Central Mindanao University.

Mishler, B. D.

1997 Major features of the evolution of bryophytes. Abstract: 22.

Pant, G. And Tewari, S. D.

1989 Various human uses of bryophytes in the Kumaun region of Northwest Himalaya. Bryologist 92: 120-122.

Saxena, D.K. and Harinder.

2004 Uses of bryophytes. Resonance. 9 (6): 56-65, D01:10.1007/ BF02839221

Shaw, A. J. and K. J. Renzaglia.

2004 Phylogeny and diversification of bryophytes. American Journal of botany 91: 1557-1581.

Sturtevant, W.

1954 The Mikasuki seminole: medical beliefs and practices. Ph. D. Dissertation. Yale University, p. 203.

Tan, B. C. and Z. Iwatsuki

1991 A new annotated Philippine moss checklist. Harvard Papers n Botany 3: 1-65.

Turner, N. J., Thomas, J., Carlson, B. F., and Ogilvie, R. T.

1983 Ethnobotany of the Nitinaht Indians of Vancouver Island. Victoria. British Columbia Provincial Museum, p. 59.

Wu, P. C.

1977 Rhodobryum giganteum(Schwaegr.) Par can be used for 
Yakubik, K.

2000 The role of bryophytes in stream ecosystems. Undergraduate Ecology Research Reports.Institute of Ecosystm Studies.

Yamaguchi, T.

1993 A revision of the genus Leucobryum(Musci) in Asia. Journal of Hattori Botany Laboratory 73: 1-123. 